\title{
Nonrelativistic Quantum Mechanics with Fundamental Environment
}

\author{
Ashot S. Gevorkyan \\ Institute for Informatics and Automation Problems, \\ National Academy of Sciences \\ BioArt Company (LTD) \\ Republic of Armenia
}

\section{Introduction}

Now it is obvious that quantum mechanics enters in the 21st century into a principally new and important phase of its development which will cardinally change the currently used technical facilities in the areas of information and telecommunication technologies, exact measurements, medicine etc. Indisputably, all this on the whole will change the production potential of human civilization and influence its morality. Despite unquestionable success of quantum physics in the 20th century, including creation of lasers, nuclear energy use, etc. it seems that possibilities of the quantum nature are not yet studied and understood deeply, a fortiori, are used.

The central question which arises on the way of gaining a deeper insight into the quantum nature of various phenomena is the establishment of well-known accepted criteria of applicability of quantum mechanics. In particular, the major of them is the de-Broglie criterion, which characterizes any body-system by a wave the length of which is defined as $\lambda=\hbar / p$, where $\lambda$ is the wavelength of the body-system, $p$ is its momentum and $\hbar$ is the Plank constant. An important consequence of this formula is that it assigns the quantum properties only to such systems which have extremely small masses. Moreover, it is well known that molecular systems which consist of a few heavy atoms are, as a rule, well described by classical mechanics. In other words, the de-Broglie criterion is an extremely strong limitation for occurrence of quantum effects in macroscopic systems. Till now only a few macroscopic quantum phenomena have been known, such as superfluidity and superconductivity, which are not ordinary natural phenomena but most likely extremal states of nature. Thus, a reasonable question arises, namely, how much correct is the deBroglie criterion, or more precisely, how completely this criterion reflects the quantum properties of a system.

In order to answer this essentially important question for development of quantum physics, it is necessary to expand substantially the concepts upon which quantum mechanics is based. The necessity for generalization of quantum mechanics is also dictated by our aspiration to consider such hard-to-explain phenomena as spontaneous transitions between the quantum levels of a system, the Lamb Shift of energy levels, EPR paradox, etc. within the limits of a united scheme. In this connection it seems important to realize finally the concept according to which any quantum system is basically an open 
system, especially when we take into account the vacuum's quantum fluctuations [1- 3]. Specifically for a quantum noise coming from vacuum fluctuations we understand a stationary Wiener-type source with noise intensity proportional to the vacuum power $P \equiv \hbar<\omega^{2}>/ 4$, where $<\omega^{2}>$ is the variance of the field frequencies averaged over some appropriate distribution (we assume $\langle\omega\rangle=0$ since $\omega$ and $-\omega$ must be considered as independent fluctuations). For example, in the cosmic background case where $T=2 \mathrm{~K}$ we find, correspondingly, $P=1.15 \mathrm{pW}$. Calculation of $\left\langle\omega^{2}\right\rangle$ for quantum fluctuations is not trivial because vacuum energy density diverges as $\omega^{3}$ [3] with uniform probability distribution denying a simple averaging process unless physical cutoffs at high frequencies exist.

Thus, first of all we need such a generalization of quantum mechanics which includes nonperturbative vacuum as fundamental environment (FE) of a quantum system (QS). As our recent theoretical works have shown [4-9], this can be achieved by naturally including the traditional scheme of nonrelativistic quantum mechanics if we define quantum mechanics in the limits of a nonstationary complex stochastic differential equation for a wave function (conditionally named a stochastic Schrödinger equation). Indeed, within the limits of the developed approach it is possible to solve the above-mentioned traditional difficulties of nonrelativistic quantum mechanics and obtain a new complementary criterion which differs from de-Broglie's criterion. But the main achievement of the developed approach is that in the case when the de-Broglie wavelength vanishes and the system, accordingly, becomes classical within the old conception, nevertheless, it can have quantum properties by a new criterion.

Finally, these quantum properties or, more exactly, quantum-field properties can be strong enough and, correspondingly, important for their studying from the point of view of quantum foundations and also for practical applications.

The chapter is composed of two parts. The first part includes a general scheme of constructing the nonrelativistic quantum mechanics of a bound system with FE. In the second part of the chapter we consider the problem of a quantum harmonic oscillator with fundamental environment. Since this model is being solved exactly, its investigation gives us a lot of new and extremely important information on the properties of real quantum systems, which in turn gives a deeper insight into the nature of quantum foundations.

\section{Formulation of the problem}

We will consider the nonrelativistic quantum system with random environment as a closed united system QS and FE within the limits of a stochastic differential equation (SDE) of Langevin-Schrödinger (L-Sch) type:

$$
i \partial_{t} \Psi_{s t c}=\hat{H}(\mathbf{x}, t ;\{\mathbf{f}\}) \Psi_{s t c}, \quad \partial_{t} \equiv \partial / \partial_{t}, \quad-\infty<t<+\infty .
$$

In equation (2.1) the stochastic operator $\hat{H}(\mathbf{x}, t ;\{\mathbf{f}\})$ describes the evolution of the united system QS + FE, where $\{\mathbf{f}\}$ is a random vector forces generating the environment fluctuations. In addition, in the units $\hbar=m=1$ the operator has the form:

$$
\hat{H}(\mathbf{x}, t ;\{\mathbf{f}\})=-\frac{1}{2} \Delta+V(\mathbf{x}, t ;\{\mathbf{f}\}), \quad \mathbf{x} \in \mathbf{R}^{3},
$$


where $\Delta$ denotes a Laplace operator, $V(\mathbf{x}, t ;\{\mathbf{f}\})$ describes the interaction potential in a quantum system which has regular and stochastic terms.

We will suppose that when $\{\mathbf{f}\} \equiv 0$, the system executes regular motion which is described by the regular nonstationary interaction potential $V_{0}(\mathbf{x}, t)=\left.V(\mathbf{x}, t ;\{\mathbf{f}\})\right|_{\{\mathbf{f}\}=0}$. In this case the quantum system will be described by the equation:

$$
i \partial_{t} \Psi=\hat{H}_{0}(\mathbf{x}, t) \Psi,\left.\quad \hat{H}_{0}(\mathbf{x}, t) \equiv \hat{H}(\mathbf{x}, t ;\{\mathbf{f}\})\right|_{\{\mathbf{f}\}=0} .
$$

We also assume that in the limit $t \rightarrow-\infty$ the QS passes to an autonomous state which mathematically equals to the problem of eigenvalues and eigenfunctions:

$$
\Delta \Phi_{-}+2\left(E_{-}-V_{-}(\mathbf{x})\right) \Phi_{-}=0,\left.\quad \Psi(\mathbf{x}, t)\right|_{t \rightarrow-\infty} \sim e^{-i E_{-} t} \Phi_{-},
$$

where in the (in) asymptotic state $E_{-}$designates the energy of the quantum system and, correspondingly, the interaction potential is defined by the limit: $V_{-}(\mathbf{x})=\lim _{t \rightarrow-\infty} V_{0}(\mathbf{x}, t)$. In the (out) asymptotic state when the interaction potential tends to the limit: $V_{+}=\lim _{t \rightarrow+\infty} V_{0}(\mathbf{x}, t)$, the QS is described by the orthonormal basis $\left\{\Phi_{+}(\mathbf{g} \mid \mathbf{x})\right\}$ and eigenvalues $\left\{E_{+} \mathbf{g}\right\}$, where $\mathbf{g} \equiv(n, m, \ldots)$ designates an array of quantum numbers.

Further we assume that the solution of problem (2.4) leads to the discrete spectrum of energy and wave functions which change adiabatically during the evolution (problem (2.3)). The latter implies that the wave functions form a full orthogonal basis:

$$
\int_{\mathbf{R}^{3}} \Psi(\mathbf{g} \mid \mathbf{x}, t) \Psi^{*}\left(\mathbf{g}^{\prime} \mid \mathbf{x}, t\right) d^{3} \mathbf{x}=\delta_{\mathbf{g}^{\prime} \mathbf{g}^{\prime}}
$$

where the symbol * means complex conjugation.

Finally, it is important to note that an orthogonality condition similar to (2.5) can be written also for a stochastic wave function: $\int_{R^{3}} \Psi_{s t c}(\mathbf{g} \mid \mathbf{x}, t ;\{\xi\}) \Psi^{*}\left(\mathbf{g}^{\prime} \mid \mathbf{x}, t ;\{\xi\}\right) d^{3} \mathbf{x}=1$, where $\{\xi\}$ designates random field (definition see below).

\subsection{The equation of environment evolution}

The solution of (2.1) can be represented,

$$
\Psi_{s t c}(\mathbf{x}, t ;\{\xi\})=\sum_{\mathbf{g}} U_{\mathbf{g}}(t) \Psi(\mathbf{g} \mid \mathbf{x}, t) .
$$

Now substituting (2.6) into (2.1) with taking into account (2.3) and (2.5), we can find the following system of complex SDEs:

$$
i \delta_{\mathbf{g}^{\prime} \mathbf{g}} \dot{U}_{\mathbf{g}^{\prime}}(t)=\left\{i A_{\mathbf{g}^{\prime} \mathbf{g}}(t)+F_{\mathbf{g}^{\prime} \mathbf{g}}(t ;\{\mathbf{f}\})\right\} U_{\mathbf{g}^{\prime}}, \quad \dot{U}_{\mathbf{g}}(t)=d U_{\mathbf{g}}(t) / d t,
$$

where the following designations are made:

$$
\begin{aligned}
& A_{\mathbf{g}^{\prime} \mathbf{g}}(t)=\int_{\mathbf{R}^{3}} \Psi^{*}(\mathbf{g} \mid \mathbf{x}, t) \partial_{t} \Psi\left(\mathbf{g}^{\prime} \mid \mathbf{x}, t\right) d^{3} \mathbf{x}, \\
& F_{\mathbf{g}^{\prime} \mathbf{g}}(t ;\{\mathbf{f}\})=\int_{\mathbf{R}^{3}} \Psi^{*}(\mathbf{g} \mid \mathbf{x}, t)\left[V(\mathbf{x}, t ;\{\mathbf{f}\})-V_{0}(\mathbf{x}, t)\right] \Psi\left(\mathbf{g}^{\prime} \mid \mathbf{x}, t\right) d^{3} \mathbf{x} .
\end{aligned}
$$


Recall that in (2.7) dummy indices denote summations; in addition, it is obvious that the coefficients $A_{\mathbf{g}^{\prime} \mathbf{g}}(t)$ and $F_{\mathbf{g}^{\prime} \mathbf{g}}(t ;\{\mathbf{f}\})$ are, in general, complex functions.

For further investigations it is useful to represent the function $U_{\mathbf{g}}(t)$ in the form of a sum of real and imaginary parts:

$$
U_{\mathbf{g}}(t)=u_{\mathbf{g}}(t)+i v_{\mathbf{g}}(t)
$$

Now, substituting expression (2.8) into (2.7), we can find the following system of SDEs:

$$
\left\{\begin{array}{l}
\delta_{\mathbf{g}^{\prime} \mathbf{g}} \dot{u}_{\mathbf{g}^{\prime}}=\left[A_{\mathbf{g}^{\prime} \mathbf{g}}^{(1)}(t)+F_{\mathbf{g}^{\prime} \mathbf{g}}^{(2)}(t ;\{\mathbf{f}\})\right] u_{\mathbf{g}^{\prime}}-\left[A_{\mathbf{g}^{\prime} \mathbf{g}}^{(2)}(t)-F_{\mathbf{g}^{\prime} \mathbf{g}}^{(1)}(t ;\{\mathbf{f}\})\right] v_{\mathbf{g}^{\prime}{ }^{\prime}} \\
\delta_{\mathbf{g}^{\prime} \mathbf{g}} \dot{v}_{\mathbf{g}^{\prime}}=\left[A_{\mathbf{g}^{\prime} \mathbf{g}}^{(2)}(t)-F_{\mathbf{g}^{\prime} \mathbf{g}}^{(1)}(t ;\{\mathbf{f}\})\right] u_{\mathbf{g}^{\prime}}+\left[A_{\mathbf{g}^{\prime} \mathbf{g}}^{(1)}(t)+F_{\mathbf{g}^{\prime} \mathbf{g}}^{(2)}(t ;\{\mathbf{f}\})\right] v_{\mathbf{g}^{\prime}}
\end{array}\right.
$$

where the following designations are made:

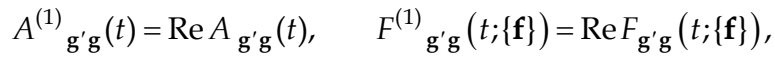

$$
\begin{aligned}
& A^{(2)} \mathbf{g}^{\prime} \mathbf{g}(t)=\operatorname{Im} A_{\mathbf{g}^{\prime} \mathbf{g}}(t), \quad F^{(2)}{ }_{\mathbf{g}^{\prime} \mathbf{g}}(t ;\{\mathbf{f}\})=\operatorname{Im} F_{\mathbf{g}^{\prime} \mathbf{g}}(t ;\{\mathbf{f}\}) \text {. }
\end{aligned}
$$

Ordering a set of random processes $\left\{u_{\mathbf{g}}(t), v_{\mathbf{g}}(t)\right\}$, the coefficients $\left\{A^{(1)} \mathbf{g}^{\prime} \mathbf{g}(t), A^{(2)} \mathbf{g}^{\prime} \mathbf{g}(t)\right\}$ and random forces $\left\{F_{\mathrm{gg}^{\prime}}{ }^{(1)}(t ;\{\mathbf{f}\}), F_{\mathrm{gg}}{ }^{(2)}(t ;\{\mathbf{f}\})\right\}$, one can rewrite the system of SDEs as:

$$
\dot{\xi}_{i}=a_{i}(\xi, t)+\sum_{j=1}^{n} b_{i j}(\xi, t) f_{j}(t), \quad \dot{\xi}_{i}(t)=d \xi_{i} / d t .
$$

In the system of equations (2.10) the symbol $\xi$ describes a random vector process represented in the following form: $\xi \equiv \boldsymbol{\xi}\left(\ldots u_{g_{i}} \ldots, \ldots v_{g_{j}} \ldots\right), \quad(1, \ldots i \ldots, j \ldots, n)$, where $n$ is the total number of random components which is twice as big as the total number of quantum states. In addition, the members $a_{i}(\xi, t)$ in equations (2.10) are composed of the matrix elements $\left\{A^{(1)} \mathbf{g}^{\prime} \mathbf{g}(t), A^{(2)} \mathbf{g}^{\prime} \mathbf{g}(t)\right\}$ and regular parts of matrix elements $\left\{F_{\mathbf{g}^{\prime} \mathbf{g}}^{(1)}(t ;\{\mathbf{f}\}), F^{(2)} \mathbf{g}^{\prime} \mathbf{g}(t ;\{\mathbf{f}\})\right\}$ while the random forces $f_{j}(t)$ are composed of random parts of the above matrix elements.

Assuming that random forces satisfy the conditions of white noise:

$$
\left\langle f_{j}(t)\right\rangle=0, \quad\left\langle f_{i}(t) f_{j}\left(t^{\prime}\right)\right\rangle=\lambda_{i j} \delta\left(t-t^{\prime}\right),
$$

where $\lambda_{i j}=0$, if $i \neq j$ and $\lambda_{i i} \equiv \lambda_{i}>0$.

Now, using the system of equations (2.10) and correlation properties (2.11), it is easy to obtain the Fokker-Planck equation for the joint probability distribution of fields $\{\xi\}$ (see in particular $[6,10])$ :

$$
\partial_{t} P=\hat{L}^{(n)} P,
$$

where the operator $\hat{L}^{(n)}$ is defined by the form: 


$$
\hat{L}^{(n)}=-\sum_{i=1}^{n} \frac{\partial}{\partial \xi_{i}} a_{i}(\boldsymbol{\xi}, t)+\sum_{i, j, k, l=1}^{n} \frac{\partial}{\partial \xi_{i}}\left(b_{i k}(\boldsymbol{\xi}, t) \frac{\partial}{\partial \xi_{j}} b_{j l}(\boldsymbol{\xi}, t)\right),
$$

The joint probability in (2.12) is defined by the expression:

$$
P\left(\mathcal{\xi}, t \mid \boldsymbol{\xi}_{0}, t_{0}\right)=\left\langle\prod_{i=1}^{n} \delta\left(\xi_{i}(t)-\xi_{0 i}\right)\right\rangle, \quad \xi_{0 i} \equiv \xi_{i}\left(t_{0}\right),
$$

From this definition, in particular, it follows that equation (2.12) must satisfies to the initial condition:

$$
\left.P\left(\xi, t \mid \xi_{0}, t_{0}\right)\right|_{t=t_{0}}=\prod_{i=1}^{n} \delta\left(\xi_{i}-\xi_{0 i}\right),
$$

where $t_{0}$ is the moment of switching of environment influence; in addition, the coordinates $\xi_{i}$ compose the $n$-dimensional non-Euclidian space $\xi_{i} \in \mathbf{\Xi}^{n}$.

Finally, since the function $P\left(\xi, t \mid \xi_{0}, t_{0}\right)$ has the meaning of probability distribution, we can normalize it:

$$
\int_{\Xi^{n}} \tilde{P}\left(\xi, t \mid \xi_{0}, t_{0}\right) d^{n} \boldsymbol{\xi}=1, \quad \tilde{P}\left(\mathcal{\xi}, t \mid \xi_{0}, t_{0}\right)=N^{-1}(t) P\left(\xi, t \mid \xi_{0}, t_{0}\right),
$$

where the function $N(t)$ is the term which implements performing of the normalization condition to unit, defined by the expression: $N(t)=\int_{\Xi^{(n)}} P\left(\xi, t \mid \xi_{0}, t_{0}\right) d^{n} \boldsymbol{\xi}$.

\subsection{Stochastic density matrix method}

We consider the following bilinear form (see representation (2.6)):

$$
\rho_{s t c}\left(\mathbf{x}, t ;\{\xi\} \mid \mathbf{x}^{\prime}, t^{\prime} ;\left\{\xi^{\prime}\right\}\right)=\sum_{\mathbf{g} \mathbf{g}^{\prime}} U_{\mathbf{g}}(t) U_{\mathbf{g}^{\prime}}^{*}\left(t^{\prime}\right) \Psi(\mathbf{g} \mid \mathbf{x}, t) \Psi^{*}\left(\mathbf{g}^{\prime} \mid \mathbf{x}^{\prime}, t^{\prime}\right),
$$

where the symbol "*" means complex conjugation.

After integrating (2.15) by the coordinates $\mathbf{x} \in \mathbf{R}^{3}$ and $\xi \in \boldsymbol{\Xi}^{n}$ with taking into account the weight function (2.13), we can find:

$$
I(t)=\operatorname{Tr}_{\xi} \operatorname{Tr}_{\mathbf{x}}\left[\rho_{s t c}\left(\mathbf{x}, t ;\{\xi\} \mid \mathbf{x}^{\prime}, t^{\prime} ;\left\{\xi^{\prime}\right\}\right)\right]=\sum_{\mathbf{g}}\left\langle\left|U_{\mathbf{g}}(t)\right|^{2}\right\rangle,
$$

where $\left\langle\left|U_{\mathbf{g}}(t)\right|^{2}\right\rangle=\operatorname{Tr}_{\xi}\left\{\left|U_{\mathbf{g}}(t)\right|^{2}\right\}=\int_{\Xi^{n}} \tilde{P}\left(\xi, t \mid \xi_{0}, t_{0}\right)\left|U_{\mathbf{g}}(t)\right|^{2} d^{n} \xi$.

Now, using (2.16) we can construct an expression for a usual nonstationary density matrix [12]:

$$
\rho\left(\mathbf{x}, t \mid \mathbf{x}^{\prime}, t^{\prime}\right)=\sum_{\mathbf{g}} \Lambda_{\mathbf{g}}(t) \Psi(\mathbf{g} \mid \mathbf{x}, t) \Psi^{*}\left(\mathbf{g}^{\prime} \mid \mathbf{x}^{\prime}, t^{\prime}\right) .
$$


where $\Lambda_{\mathbf{g}}(t)=\left\langle\left|U_{\mathbf{g}}(t)\right|^{2}\right\rangle / I(t)$ has the meaning of level population of the quantum state under the conditions of equilibrium between the quantum system and fundamental environment. It is easy to check that the stochastic density matrix $\rho_{\text {stc }}\left(\mathbf{x}, t ;\{\boldsymbol{\xi}\} \mid \mathbf{x}^{\prime}, t^{\prime} ;\left\{\mathcal{\zeta}^{\prime}\right\}\right)$ satisfy to von Neumann equation while the reduced density matrix $\rho\left(\mathbf{x}, t \mid \mathbf{x}^{\prime}, t^{\prime}\right)$ does not satisfies the equation. Taking into account equations (2.1), (2.13) and (2.15), we can obtain the evolution equation for reduced density matrix:

$$
i\left\langle\partial_{t} \rho_{s t c}\left(\mathbf{x}, t ;\{\xi\} \mid \mathbf{x}^{\prime}, t^{\prime} ;\left\{\mathcal{\zeta}^{\prime}\right\}\right)\right\rangle=\left\langle\left[\hat{H}, \rho_{s t c}\left(\mathbf{x}, t ;\{\xi\} \mid \mathbf{x}^{\prime}, t^{\prime} ;\left\{\mathcal{\zeta}^{\prime}\right\}\right)\right]\right\rangle .
$$

where $\operatorname{Tr}_{\xi}\{\ldots\}=\langle\ldots\rangle$, in addition $[\ldots]$ describes the quantum Poisson brackets which denote the commentator: $[A, B]=A B-B A$.

It is obvious that equation (2.18) is a nonlocal equation. Taking into account (2.12), one can bring equation (2.18) to the form:

$$
i \partial_{t} \rho\left(\mathbf{x}, \mathbf{x}^{\prime}, t\right)=\left[\hat{H}_{0}, \rho\left(\mathbf{x}, \mathbf{x}^{\prime}, t\right)\right]+i \int_{\Xi^{n}} \rho_{s t c}\left(\mathbf{x}, \mathbf{x}^{\prime}, t ;\{\xi\}\right) \hat{L}^{(n)} \tilde{P}\left(\boldsymbol{\xi}, t \mid \xi_{0}, t_{0}\right) d^{n} \boldsymbol{\xi},
$$

where following designations are made; $\rho\left(\mathbf{x}, \mathbf{x}^{\prime}, t\right)=\left.\rho\left(\mathbf{x}, t \mid \mathbf{x}^{\prime}, t^{\prime}\right)\right|_{t=t^{\prime}}$ is a reduced density matrix, in addition, $\rho_{\text {stc }}\left(\mathbf{x}, \mathbf{x}^{\prime}, t ;\{\xi\}\right)=\left.\rho_{s t c}\left(\mathbf{x}, t ;\{\xi\} \mid \mathbf{x}^{\prime}, t^{\prime} ;\left\{\mathcal{\xi}^{\prime}\right\}\right)\right|_{t=t^{\prime}}$.

Thus, equation (2.19) differs from the usual von Neumann equation for the density matrix. The new equation (2.19), unlike the von Neumann equation, considers also the exchange between the quantum system and fundamental environment, which in this case plays the role of a thermostat.

\subsection{Entropy of the quantum subsystem}

For a quantum ensemble, entropy was defined for the first time by von Neumann [11]. In the considered case where instead of a quantum ensemble one united system QS + FE, the entropy of the quantum subsystem is defined in a similar way:

$$
S(\boldsymbol{\lambda} ; t)=-\operatorname{Tr}_{\mathbf{x}}\left[\rho\left(\mathbf{x}, \mathbf{x}^{\prime}, t\right) \ln \rho\left(\mathbf{x}, \mathbf{x}^{\prime}, t\right)\right], \quad \lambda=\left\{\lambda_{i}\right\} .
$$

In connection with this, there arises an important question about the behavior of the entropy of a multilevel quantum subsystem on a large scale of times. It is obvious that the relaxation process can be nontrivial (for example, absence of the stationary regime in the limit $t \rightarrow+\infty)$ and, hence, its investigation will be a difficult-to-solve problem both by analytic methods and numerical simulation.

A very interesting case is when the QS breaks up into several subsystems. In particular, when the QS breaks up into two fragments and when these fragments are spaced far from each other, we can write for a reduced density matrix of the subsystem the following expression:

$$
\rho\left(\mathbf{x}, \mathbf{x}^{\prime}, t\right)=\rho_{1}\left(\mathbf{y}, \mathbf{y}^{\prime}, t\right) \rho_{2}\left(\mathbf{z}, \mathbf{z}^{\prime}, t\right), \quad \mathbf{x} \equiv \mathbf{x}(\mathbf{y} ; \mathbf{z}), \quad(\mathbf{y} ; \mathbf{z}) \in R^{3} .
$$

Recall that the vectors $\mathbf{y}$ and $\mathbf{z}$ describe the first and second fragments, correspondingly. Now, substituting the reduced density matrix $\rho\left(\mathbf{x}, \mathbf{x}^{\prime}, t\right)$ into the expression of the entropy of QS (2.20), we obtain:

$$
S(\boldsymbol{\lambda} ; t)=-J_{1}(\boldsymbol{\lambda} ; t) S_{2}(\boldsymbol{\lambda} ; t)-J_{2}(\boldsymbol{\lambda} ; t) S_{1}(\boldsymbol{\lambda} ; t)
$$


where the following designations are made in expression (2.22):

$$
\begin{gathered}
J_{1}(\boldsymbol{\lambda} ; t)=\operatorname{Tr}_{\mathbf{y}}\left[\rho_{1}\left(\mathbf{y}, \mathbf{y}^{\prime}, t\right)\right], S_{1}(\boldsymbol{\lambda} ; t)=-\operatorname{Tr}_{\mathbf{y}}\left[\rho\left(\mathbf{y}, \mathbf{y}^{\prime}, t\right) \ln \rho\left(\mathbf{y}, \mathbf{y}^{\prime}, t\right)\right], \\
J_{2}(\boldsymbol{\lambda} ; t)=\operatorname{Tr}_{\mathbf{z}}\left[\rho_{2}\left(\mathbf{z}, \mathbf{z}^{\prime}, t\right)\right], S_{2}(\boldsymbol{\lambda} ; t)=-\operatorname{Tr}_{\mathbf{z}}\left[\rho\left(\mathbf{z}, \mathbf{z}^{\prime}, t\right) \ln \rho\left(\mathbf{z}, \mathbf{z}^{\prime}, t\right)\right] .
\end{gathered}
$$

Since at the beginning of evolution the two subsystems interact with each other, it is easy to show that $J_{1}(\lambda ; t) \neq 1$ and $J_{2}(\lambda ; t) \neq 1$, moreover, they can be fluctuated depending on the time. The last circumstance proves that the subsystems of the QS are in the entangled state. This means that between the two subsystems there arises a new type of nonpotential interaction which does not depend on the distance and size of the subsystems. In the case when subsystems 1 and 2 have not interacted, $J_{1}=J_{2}=1$ and, correspondingly, $S_{1}$ and $S_{2}$ are constants denoting entropies of isolated systems.

\subsection{Conclusion}

The developed approach allows one to construct a more realistic nonrelativistic quantum theory which includes fundamental environment as an integral part of the quantum system. As a result, the problems of spontaneous transitions (including decay of the ground state) between the energy levels of the QS, the Lamb shift of the energy levels, ERP paradox and many other difficulties of the standard quantum theory are solved naturally. Equation (2.12) - $\left(2.13^{\prime}\right)$ describes quantum peculiarities of FE which arises under the influence of the quantum system. Unlike the de-Broglie wavelength, they do not disappear with an increase in mass of the quantum subsystem. In other words, the macroscopic system is obviously described by the classical laws of motion; however, space-times structures can be formed in FE under its influence. Also, it is obvious that these quantum-field structures ought to be interpreted as a natural continuation and addition to the considered quantum (classical) subsystem. These quantum-field structures under definite conditions can be quite observable and measurable. Moreover, it is proved that after disintegration of the macrosystem into parts its fragments are found in the entangled state, which is specified by nonpotential interaction (2.22), and all this takes place due to fundamental environment. Especially, it concerns nonstationary systems, for example, biological systems in which elementary atom-molecular processes proceed continuously [13]. Note that such a conclusion becomes even more obvious if one takes into account the well-known work [14] where the idea of universal description for unified dynamics of micro- and macroscopic systems in the form of the Fokker-Planck equation was for the first time suggested.

Finally, it is important to add that in the limits of the developed approach the closed system QS + FE in equilibrium is described in the extended space $R^{3} \otimes \Xi^{n}$, where $\Xi^{n}$ can be interpreted as a compactified subspace in which FE in equilibrium state is described.

\section{The quantum one-dimensional harmonic oscillator (QHO) with FE as a problem of evolution of an autonomous system on the stochastic space-time continuum}

As has been pointed out in the first part of the chapter, there are many problems of great importance in the field of non-relativistic quantum mechanics, such as the description of the Lamb shift, spontaneous transitions in atoms, quantum Zeno effect [15] etc., which remain 
unsolved due to the fact that the concept of physical vacuum has not been considered within the framework of standard quantum mechanics. There are various approaches for investigation of the above-mentioned problems: the quantum state diffusion method [16], Lindblad density matrix method [17, 18], quantum Langevin equation [19], stochastic Schrödinger equation method (see [12]), etc. Recall that representation [17, 18] describes a priori the most general situation which may appear in a non-relativistic system. One of these approaches is based on the consideration of the wave function as a random process, for which a stochastic differential equation (SDE) is derived. However, the consideration of a reduced density matrix on a semi-group [20] is quite an ambiguous procedure and, moreover, its technical realization is possible, as a rule, only by using the perturbation method. For investigation of the inseparably linked closed system QSE, a new mathematical scheme has been proposed [5-8] which allows one to construct all important parameters of the quantum system and environment in a closed form. The main idea of the developed approach is the following. We suppose that the evolution time of the combined system consists of an infinite set of time intervals with different duration, where at the end of each interval a random force generated by the environment influences the quantum subsystem. At the same time the motion of the quantum subsystem within each time interval can be described by the Schrödinger equation. Correspondingly, the equation which describes the combined closed system QSE on a large scale of time can be represented by the stochastic differential equation of Langevin-Schrödinger (L-Sch) type.

In this section, within the framework of the 1D L-Sch equation an exact approach for the quantum harmonic oscillator $(\mathrm{QHO})$ model with fundamental environment is constructed. In particular, the method of stochastic density matrix (SDM) is developed, which permits to construct all thermodynamic potentials of the quantum subsystem analytically, in the form of multiple integrals from the solution of a $2 \mathrm{D}$ second-order partial differential equation.

\subsection{Description of the problem}

We will consider that the $1 \mathrm{D} \mathrm{QHO}+\mathrm{FE}$ closed system is described within the framework of the L-Sch type SDE (see equation (2.1)), where the evolution operator has the following form:

$$
\hat{H}(x, t ;\{f\})=\frac{1}{2}\left[-\partial_{x}^{2}+\Omega^{2}(t ;\{f\}) x^{2}\right], \quad-\infty<x<+\infty .
$$

In expression (3.1) the frequency $\Omega(t ;\{\mathbf{f}\})$ is a random function of time where its stochastic component describes the influence of environment. For the analysis of a model of an environment a set of harmonic oscillators [21-25] and quantized field [26, 27] are often used. For simplicity, we will assume that frequency has the following form:

$$
\Omega^{2}(t ;\{f\})=\Omega_{0}^{2}+f(t), \quad \Omega_{0}=\text { const }, \quad \lim _{t \rightarrow-\infty} f(t)=0,
$$

where $f(t)$ is an independent Gaussian stochastic process with a zero mean and $\delta$ is a shaped correlation function:

$$
\langle f(t)\rangle=0, \quad\left\langle f(t) f\left(t^{\prime}\right)\right\rangle=2 \lambda \delta\left(t-t^{\prime}\right) .
$$


The constant of $\lambda$ characterizes power of stochastic force $f(t)$. Equation (2.1) with operator (3.1) has an asymptotic solution $\Psi(n \mid x, t)$ in the limit $t \rightarrow-\infty$ :

$$
\Psi(n \mid x, t)=e^{-i(n+1 / 2) \Omega_{0} t} \phi(n \mid x), \quad \phi(n \mid x)=\left[\frac{1}{2^{n} n !} \sqrt{\frac{\Omega_{0}}{\pi}}\right]^{1 / 2} e^{-\Omega_{0} x^{2} / 2} H_{n}\left(\sqrt{\Omega_{0}} x\right),
$$

where $n=0,1,2 \ldots$ in addition; $\phi(n \mid x)$ is the wave function of a stationary oscillator and $H_{n}(y)$ is the Hermitian polynomial. The formal solution of problem (2.1), (3.1)-(3.4) may be written down explicitly for arbitrary $\Omega(t ;\{f\}$ ) (see [28]). It has the following form:

$$
\Psi_{s t c}(x, t \mid\{\xi\})=\frac{1}{\sqrt{r(t)}} \exp \left\{\frac{i}{2} \frac{r_{t}(t)}{r(t)} x^{2}\right\} \chi\left(\frac{x}{r(t)}, \tau\right), \quad \xi=r(t) e^{i \gamma(t)}, \quad r_{t}(t)=\frac{d r(t)}{d t},
$$

where the function $\chi(y, \tau)$ describes the wave function of the Schrödinger equation:

$$
i \partial_{\tau} \chi=\frac{1}{2}\left[-\partial_{y}^{2}+\Omega_{0}^{2} y^{2}\right] \chi,
$$

for a harmonic oscillator on the stochastic space-time $\{y, \tau\}$ continuum. In (3.6) the following designations are made:

$$
y=x / r, \quad \tau=\gamma(t) / \Omega_{0}, \quad \gamma(t)=\int_{-\infty}^{t} d t^{\prime} / r^{2}\left(t^{\prime}\right)
$$

The random solution $\xi(t)$ satisfies the classical homogeneous equation of an oscillator which describes the stochastic fluctuating process flowing into FE:

$$
\ddot{\xi}+\Omega^{2}(t ;\{f\}) \xi=0
$$

Taking into account (3.5) and the well-known solution of autonomous quantum harmonic oscillator (3.6) (see [28]) for stochastic complex processes which describe the 1D QHO+FE closed system, we can write the following expression:

$$
\begin{aligned}
& \Psi_{s t c}(n|x, t|\{\xi\})= \\
& =\left[\frac{1}{2^{n} n !} \sqrt{\frac{\Omega_{0}}{\pi r}}\right]^{1 / 2} \exp \left\{-i\left(n+\frac{1}{2}\right) \Omega_{0} \int_{t_{0}}^{t} \frac{d t^{\prime}}{r^{2}\left(t^{\prime}\right)}+\frac{1}{2}\left(i \frac{r_{t}}{r}-\frac{\Omega_{0}}{r^{2}}\right) x^{2}\right\} H_{n}\left(\sqrt{\Omega_{0}} \frac{x}{r}\right) .
\end{aligned}
$$

The solution of (3.8) is defined in the extended space $\Xi=R^{1} \otimes R_{\{\xi\}}$, where $R^{1}$ is the onedimensional Euclidian space and $R_{\{\xi\}}$ is the functional space which will be defined below (see section 3.3). Note that wave function (3.8) (a more specific wave functional) describes the quantum subsystem with taking into account the influence of the environment. It is easy to show that complex probabilistic processes (3.8) consist of a full orthogonal basis in the space of quadratically integrable functions $L_{2}$. 
Taking into account the orthogonal properties of (3.8), we can write the following normalization condition:

$$
\int_{-\infty}^{+\infty} \Psi_{s t c}(n|x, t|\{\xi\}) \Psi_{s t c}^{*}(m|x, t|\{\xi\}) d x=\delta_{n m},
$$

where the symbol "*" means complex conjugation.

So, the initial L-Sch equation (2.1) - (3.1) which satisfies the asymptotic condition (3.4) is reduced to autonomous Schrödinger equation (3.6) in the stochastic space-time using the etalon differential equation (3.7). Note that equation (3.7) with taking into account conditions (3.2) and (3.3) describes the motion of FE.

\subsection{The mean values of measurable parameters of 1D QHO}

For investigation of irreversible processes in quantum systems the non-stationary density matrix representation based on the quantum Liouville equation is often used. However, the application of this representation has restrictions [11]. It is used for the cases when the system before switching on the interaction was in the state of thermodynamic equilibrium and after switching on its evolution is adiabatic. Below, in the frames of the considered model the new approach is used for the investigation of the statistical properties of an irreversible quantum system without any restriction on the quantities and rate of interaction change. Taking into account definition (2.15), we can develop SDM method in the framework of which it is possible to calculate various measurable physical parameters of a quantum subsystem.

Definition 1. The expression for a stochastic function:

$$
\rho_{s t c}\left(x, t|\{\xi\}| x^{\prime}, t^{\prime} \mid\left\{\xi^{\prime}\right\}\right)=\sum_{m=1}^{\infty} w^{(m)} \rho_{s t c}^{(m)}\left(x, t|\{\xi\}| x^{\prime}, t^{\prime} \mid\left\{\xi^{\prime}\right\}\right)
$$

will be referred to as stochastic density matrix. Recall that the partial SDM $\rho_{s t c}^{(m)}\left(x, t|\{\xi\}| x^{\prime}, t^{\prime} \mid\left\{\xi^{\prime}\right\}\right)$ is defined by the expression: $\rho_{\text {stc }}^{(m)}\left(x, t|\{\xi\}| x^{\prime}, t^{\prime} \mid\left\{\xi^{\prime}\right\}\right)=\Psi_{\text {stc }}(m|x, t|\{\xi\}) \Psi_{\text {stc }}^{*}\left(m|x, t|\left\{\xi^{\prime}\right\}\right)$. In addition, $w^{(m)}$ describes the level of population with the energy $E_{m}=(n+1 / 2) \Omega_{0}$ until the moment of time $t_{0}$ when the random excitations of FE are turned on. Integrating (3.10) over the Euclidean space $R^{(1)}$ with taking into account (3.9), we obtain the normalization condition for weight functions:

$$
\sum_{m=1}^{\infty} w^{(m)}=1, \quad w^{(m)}>0
$$

Below we define the mean values of various operators. Note that at averaging over the extended space $\Xi$ the order of integration is important. In the case when the integral from the stochastic density matrix is taken at first in the space, $R^{1}$ and then in the functional space, $R_{\{\xi\}}$ the result becomes equal to unity. This means that in the extended space $\Xi$ all conservation laws are valid, in other words, the stochastic density matrix in this space is unitary. In the case when we take the integration in the inverse order, we get another 
picture. After integration over, $R_{\{\xi\}}$ the obtained density matrix describes quantum processes in the Euclidean space, $R^{1}$. Its trace is, in general, not unitary, which means that the conservation laws, generally speaking, can be invalid in the Euclidean space.

Definition 2. The expected value of the operator $\hat{A}(x, t \mid\{\xi\})$ in the quantum state $m$ is defined by the expression:

$$
A_{m}(\gamma)=\lim _{t \rightarrow+\infty}\left\{N_{m}^{-1}(t) \operatorname{Tr}_{x}\left[\operatorname{Tr}_{\xi} \hat{A} \rho_{s t c}^{(m)}\left(x, t|\{\xi\}| x^{\prime}, t^{\prime} \mid\left\{\xi^{\prime}\right\}\right)\right]\right\}, \quad N_{m}(t)=\operatorname{Tr}_{x}\left[\operatorname{Tr}_{\xi} \rho_{s t c}^{(m)}\right]
$$

The mean value of the operator $\hat{A}(x, t \mid\{\xi\})$ over all quantum states, respectively, will be:

$$
\bar{A}(\gamma)=\lim _{t \rightarrow+\infty}\left\{N^{-1}(t) \operatorname{Tr}_{x}\left[\operatorname{Tr}_{\xi} \hat{A} \rho_{s t c}\left(x, t|\{\xi\}| x^{\prime}, t^{\prime} \mid\left\{\xi^{\prime}\right\}\right)\right]\right\}, \quad N(t)=\operatorname{Tr}_{x}\left[\operatorname{Tr}_{\xi} \rho_{s t c}\right]
$$

Note that the operation $\mathrm{Tr}_{\xi}$ in (3.12) and (3.13) denotes functional integration:

$$
\operatorname{Tr}_{\xi}\left[K\left(x, t|\{\xi\}| x^{\prime}, t^{\prime} \mid\left\{\xi^{\prime}\right\}\right)\right]=\sqrt{\Omega_{0} / \pi} \int K\left(x, t|\{\xi\}| x^{\prime}, t^{\prime} \mid\left\{\xi^{\prime}\right\}\right) D \mu(\xi),
$$

where $D \mu(\xi)$ designates the measure of functional space which will be defined below.

If we wish to derive an expression describing the irreversible behavior of the system, it is necessary to change the definition of entropy. Let us remind that the von Neumann nonstationary entropy (the measure of randomness of a statistical ensemble) is defined by the following form:

$$
\Lambda_{N}(\gamma, t)=-\operatorname{Tr}_{x}\{\rho \ln \rho\}, \quad N(\gamma)=\lim _{t \rightarrow+\infty} N(\gamma, t),
$$

where $\rho\left(x, x^{\prime} ; t\right)=\operatorname{Tr}_{\xi}\left\{\rho_{\text {stc }}\right\}$ is a reduced density matrix, $\gamma=\Omega_{0} / \lambda^{1 / 3}$ is an interaction parameter between the quantum subsystem and environment.

Let us note that the definition of the von Neumann entropy (3.15) is correct for the quantum information theory and agrees well with the Shannon entropy in the classical limit.

Definition 3. For the considered system of 1D QHO with FE the entropy is naturally defined by the form:

$$
\Lambda_{G}(\gamma, t)=-\operatorname{Tr}_{x}\left\{\operatorname{Tr}_{\{\xi\}}\left[\rho_{s t c} \ln \rho_{s t c}\right]\right\}, \quad \Lambda_{G}(\gamma)=\lim _{t \rightarrow+\infty} \Lambda_{G}(\gamma, t),
$$

where the following designation $\rho_{s t c} \equiv \rho_{s t c}\left(x, x^{\prime}, t ;\{\xi\}\right)$ is made.

Finally, it is important to note that the sequence of integrations first in the functional space, $R_{\{\xi\}}$ and then in the Euclidean space, $R^{1}$ corresponds to non-unitary reduction of the vector's state (or non-unitary influence on the quantum subsystem).

\subsection{Derivation of an equation for conditional probability of fields. Measure of functional space $R_{\{\xi\}}$}

Let us consider the stochastic equation (3.7). We will present the solution of the equation in the following form: 


$$
\xi(t)= \begin{cases}\xi_{0}(t)=\exp \left(i \Omega_{0} t\right), & t \leq t_{0}, \\ \xi_{0}\left(t_{0}\right) \exp \left(\int_{t_{0}}^{t} \mathbf{\eta}\left(t^{\prime}\right) d t^{\prime}\right), & t>t_{0} .\end{cases}
$$

After substitution of (3.17) into (3.7) we can define the following nonlinear SDE:

$$
\dot{\mathbf{\eta}}+\mathbf{\eta}^{2}+\Omega_{0}^{2}+f(t)=0, \quad \mathbf{\eta}\left(t_{0}\right)=i \dot{\xi}_{0}\left(t_{0}\right) / \xi_{0}\left(t_{0}\right)=i \Omega_{0}, \quad \dot{\mathbf{\eta}}=\partial_{t} \mathbf{\eta} .
$$

The second equation in (3.18) expresses the condition of continuity of the function $\xi(t)$ and its first derivative at the moment of time $t=t_{0}$. Using the fact that the function $\eta(t)$ describes a complex-valued random process, the SDE (3.18) may be presented in the form of two SDE for real-valued fields (random processes). Namely, introducing the real and imaginary parts of $\boldsymbol{\eta}(t)$ :

$$
\mathbf{\eta}(t)=u_{1}(t)+i u_{2}(t), \quad u_{2}(t)>0,
$$

the following system of SDEs can be finally obtained for the fields $\mathbf{\eta}(t) \equiv \mathbf{\eta}\left(u_{1}, u_{2}\right)$ :

$$
\left\{\begin{array}{l}
\dot{u}_{1}=-u_{1}^{2}+u_{2}^{2}-\Omega_{0}^{2}-f(t)=0, \quad\left\{\begin{array}{l}
u_{1}\left(t_{0}\right)=\operatorname{Re}\left[\dot{\xi}_{0}\left(t_{0}\right) / \xi_{0}\left(t_{0}\right)\right]=0, \\
\dot{u}_{2}=-2 u_{1} u_{2},
\end{array} u_{2}\left(t_{0}\right)=\operatorname{Im}\left[\dot{\xi}_{0}\left(t_{0}\right) / \xi_{0}\left(t_{0}\right)\right]=\Omega_{0} .\right.
\end{array}\right.
$$

The pair of fields $\left(u_{1}, u_{2}\right)$ in this model is not independent because their evolution is influenced by the common random force $f(t)$. This means that the joint probability distribution of fields can be represented by the form:

$$
P\left(\mathbf{\eta}, t \mid \mathbf{\eta}_{0}, t_{0}\right)=\left\langle\prod_{i=1}^{2} \delta\left(u_{i}(t)-u_{0 i}\right)\right\rangle, \quad u_{0 i} \equiv u_{i}\left(t_{0}\right),
$$

which is a non-factorable function. After differentiation of functional (3.21) with respect to time and using SDEs (3.18) and correlation properties of the random force (3.3), as well as making standard calculations and reasonings (see [29,30]), we obtain for a distribution of fields the following Fokker-Planck equation:

$$
\begin{gathered}
\partial_{t} P=\hat{L}\left(\mathbf{\eta}, t \mid \mathbf{\eta}_{0}, t_{0}\right) P, \\
\hat{L}\left(\mathbf{\eta}, t \mid \mathbf{\eta}_{0}, t_{0}\right)=\lambda \frac{\partial^{2}}{\partial u_{1}^{2}}+\left(u_{1}^{2}-u_{2}^{2}+\Omega_{0}^{2}\right) \frac{\partial}{\partial u_{1}}+2 u_{1} u_{2} \frac{\partial}{\partial u_{2}}+4 u_{1},
\end{gathered}
$$

with the initial condition:

$$
\left.P\left(u_{1}, u_{2}, t\right)\right|_{t \rightarrow t_{0}}=\delta\left(u_{1}-u_{01}\right) \delta\left(u_{2}-u_{02}\right) .
$$

Thus, equation (3.22)-(3.23) describes the free evolution of FE.

Now, our purpose consists in constructing the measure of functional space, which is a necessary condition for further theoretical constructions. The solution of equation (3.22)(3.23) for small time intervals can be presented in the form: 


$$
P\left(\mathbf{\eta}, t \mid \mathbf{\eta}^{\prime}, t^{\prime}\right)=\frac{1}{2 \pi \sqrt{\lambda \Delta t}} \exp \left\{\frac{1}{2 \Delta t}\left[u_{1}-u_{1}^{\prime}-\left(u_{1}^{2}-u_{2}^{2}+\Omega_{0}^{2}(t)\right) \Delta t\right]^{2}\right\}, \quad t=t^{\prime}+\Delta t .
$$

So, we can state that the evolution of fields $\left(u_{1}, u_{2}\right)$ in the functional space $R_{\{\xi\}}$ is characterized by regular displacement with the velocity $\left(u_{1}^{2}-u_{2}^{2}+\Omega_{0}^{2}\right)$ against the background of Gaussian fluctuations with the diffusion value $\lambda$. The infinitely small displacement of the trajectory $\mathbf{\eta}(t)$ in the space $R_{\{\xi\}}$ is determined by expression [30]:

$$
\mathbf{\eta}(t+\Delta t)=\mathbf{\eta}(t)+\left(u_{1}^{2}-u_{2}^{2}+\Omega_{0}^{2}\right) \Delta t+f(t) \Delta t^{1 / 2} .
$$

As follows from expression (3.26), the trajectory is continuous everywhere, and, correspondingly, the condition $\left.\mathbf{\eta}(t+\Delta t)\right|_{\Delta t \rightarrow 0}=\mathbf{\eta}(t)$ is valid. However, expression (3.26) is undifferentiable everywhere owing to the presence of a term which is of the order $=\Delta t^{1 / 2}$. If we divide the time into small intervals, each of which being equal to $\Delta t=t / N$, where $N \rightarrow \infty$, then expression (3.25) can be interpreted as a probability of transition from $\mathbf{\eta}_{k} \equiv \mathbf{\eta}\left(t_{k}\right)$ to $\mathbf{\eta}_{k+1} \equiv \mathbf{\eta}\left(t_{k+1}\right)$ during the time $\Delta t$ in the process of Brownian motion. With consideration of the above, we can construct probability of fields' change on finite intervals of time or the measure of the space, $R_{\{\xi\}}$ (see [4]):

$$
\begin{aligned}
D \mu(\xi) \equiv D \mathbf{\eta}= & D \mu\left(\mathbf{\eta}_{0}\right) \cdot \lim _{N \rightarrow \infty}\left[d u_{1}\left(t_{k+1}\right) d u_{2}\left(t_{k+1}\right)\left(\frac{1}{2 \pi} \sqrt{\frac{N}{\lambda t}}\right)^{N}\right] \times \\
& \prod_{k=0}^{N} \exp \left\{-\frac{N}{2 \lambda t}\left[u_{1}\left(t_{k+1}\right)-u_{1}\left(t_{k}\right)-\left(u_{1}^{2}\left(t_{k+1}\right)-u_{2}^{2}\left(t_{k+1}\right)+\Omega_{0}^{2}\right) \frac{t_{k+1}}{N}\right]^{2}\right\},
\end{aligned}
$$

where $D \mu\left(\mathbf{n}_{0}\right)=\delta\left(u_{1}-u_{01}\right) \delta\left(u_{2}-u_{02}\right) d u_{1} d u_{2}$ (see condition (3.25)).

\subsection{Entropy of the ground state of 1D QHO with fundamental environment}

For simplicity we will suppose that $w^{(0)}=1$ and, correspondingly, $w^{(m)}=0$ for all quantum numbers $m>1$ (see expression (3.10) ). In this case the SDM (3.10) with consideration of expressions (3.8), (3.14) and (3.16) may be represented by the following form:

$$
\rho_{\text {stc }}^{(0)}\left(x, x^{\prime}, t \mid\{\xi\}\right)=\sqrt{\frac{\Omega_{0}}{\pi}} \mathrm{e}^{A}, \quad A=-\int_{t_{0}}^{t} u_{1}\left(t^{\prime}\right) d t^{\prime}+\frac{i}{2} u_{1}(t)\left(x^{2}-x^{\prime 2}\right)-\frac{1}{2} u_{2}(t)\left(x^{2}+x^{\prime 2}\right),
$$

where the following designation $\left.\rho_{s t c}^{(0)}\left(x, x^{\prime}, t \mid\{\xi\}\right) \equiv \rho_{s t c}^{(0)}\left(x, t,\{\xi\} \mid x^{\prime}, t^{\prime},\left\{\xi^{\prime}\right\}\right)\right|_{t=t^{\prime}}$ is made.

Now, we can calculate the reduced density matrix: $\rho^{0}\left(x, x^{\prime}, t\right)=\operatorname{Tr}_{\xi}\left\{\rho_{s t c}^{(0)}\left(x, x^{\prime}, t \mid\{\xi\}\right)\right\}$. Using expressions for the continuous measure (3.27) and stochastic density matrix (3.28) we can construct the corresponding functional integral which can be further calculated by the generalized Feynman-Kac formula (see Appendix 4.1, [6]):

$$
\rho^{(0)}\left(x, x^{\prime}, t\right)=\sqrt{\frac{\Omega_{0}}{\pi}} \int_{-\infty}^{+\infty} d u_{1} \int_{0}^{+\infty} d u_{2} Q_{0}\left(u_{1}, u_{2}, t\right) \exp \left\{\frac{i}{2} u_{1}(t)\left(x^{2}-x^{\prime 2}\right)-\frac{1}{2} u_{2}(t)\left(x^{2}+x^{\prime 2}\right)\right\},
$$


In expression (3.29) the function $Q_{0}\left(u_{1}, u_{2}, t\right)$ is a solution of the equation:

$$
\partial_{t} Q_{0}=\left[\hat{L}\left(u_{1}, u_{2}, t\right)-u_{1}\right] Q_{0},
$$

which satisfies the following initial and boundary conditions:

$$
\left.Q_{0}\left(u_{1}, u_{2}, t\right)\right|_{t=t_{0}}=\delta\left(u_{1}-u_{01}\right) \delta\left(u_{2}-u_{02}\right),\left.\quad Q_{0}\left(u_{1}, u_{2}, t\right)\right|_{\|\mathbf{n}\| \rightarrow \infty}=0 .
$$

Let us consider the expression for the entropy (3.17). Substituting (3.29) into (3.17) we can find:

$$
\Lambda_{G}^{(0)}(\gamma, t)=-\frac{1}{\sqrt{\pi}} \operatorname{Tr}_{x}\left\{\operatorname{Tr}_{\{\xi\}}\left[A\left[u_{1}(t), u_{2}(t), t \mid x, x^{\prime}\right] \mathrm{e}^{A\left[u_{1}(t), u_{2}(t), t \mid x, x^{\prime}\right]}\right]\right\} .
$$

After conducting integration in the space $R^{1}$ in (3.33), it is easy to find the expression:

$$
\Lambda_{G}^{(0)}(\gamma)=-\left.\partial_{\alpha} N_{\alpha}(t)\right|_{\alpha=0},
$$

where the following designations are made:

$$
N_{\alpha}(t)=\operatorname{Tr}_{\xi}\left[I_{\alpha}(t ;\{\xi\})\right], \quad I_{\alpha}(t ;\{\xi\})=u_{2}^{-1 / 2}(t) \exp \left[-(\alpha+1) \int_{t_{0}}^{t} u_{1}\left(t^{\prime}\right) d t^{\prime}\right]
$$

Similarly, as in the case with (3.29), using expressions (3.34) it is possible to calculate the functional trace in the expression $N_{\alpha}(t)$ :

$$
N_{\alpha}(t)=\int_{-\infty}^{+\infty} d u_{1} \int_{0}^{+\infty} d u_{2} \frac{1}{\sqrt{u_{2}}} Q_{\alpha}\left(u_{1}, u_{2}, t\right),
$$

where the function $Q_{\alpha}\left(u_{1}, u_{2}, t\right)$ is the solution of the equation:

$$
\partial_{t} Q_{\alpha}=\left[\hat{L}\left(u_{1}, u_{2}, t\right)-(\alpha+1) u_{1}\right] Q_{\alpha} .
$$

Recall that border conditions for (3.36) are similar to (3.31). Besides, if we assume that $\alpha=0$ in (3.35), we will obtain the normalization function $N_{0}(t)$. After calculation of the function $Q_{\alpha}\left(u_{1}, u_{2}, t\right)$ we can also calculate the function $D_{\alpha}\left(u_{1}, u_{2}, t\right) \equiv \partial_{\alpha} Q_{\alpha}\left(u_{1}, u_{2}, t\right)$. In particular, it is easy to obtain an equation for $D_{\alpha}\left(u_{1}, u_{2}, t\right)$ by differentiation of equation (3.36) with respect to a:

$$
\partial_{t} D_{\alpha}=\left[\hat{L}\left(u_{1}, u_{2}, t\right)-\alpha u_{1}\right] D_{\alpha},
$$

which is solved by initial and border conditions of type (3.31).

Introducing the designation $D_{0}\left(u_{1}, u_{2}, t\right)=\left.D_{\alpha}\left(u_{1}, u_{2}, t\right)\right|_{\alpha=0}$, it is possible to find the expression:

$$
N_{0 ; 0}(t)=\partial_{\alpha} N_{\alpha}(t)=\int_{-\infty}^{+\infty} d u_{1} \int_{0}^{+\infty} d u_{2} \frac{1}{\sqrt{u_{2}}} D_{0}\left(u_{1}, u_{2}, t\right) .
$$


Using (3.38) we can write the final form of the entropy of «ground state» in the limit of thermodynamics equilibrium:

$$
\Lambda_{G}^{(0)}(\gamma)=-N_{0 ; 0}(\gamma), \quad \gamma=\Omega_{0} / \lambda^{1 / 3} .
$$

It is simple to show that in the limit $\gamma \rightarrow \infty$ entropy aspires to zero.

Thus, at the reduction $\rho_{\text {stc }}\left(x, x^{\prime}, t \mid\{\xi\}\right) \rightarrow \rho\left(x, x^{\prime}, t\right)$ information in a quantum subsystem is lost, as a result of which the entropy changes, too. Let us remind that usually the entropy of a quantum subsystem at environment inclusion grows, however, in the considered case the behavior of the entropy depending on the interaction parameter $\gamma$ can be generally nontrivial.

\subsection{Energy spectrum of a quantum subsystem}

The energy spectrum is an important characteristic of a quantum system. In the considered case we will calculate the first two levels of the energy spectrum in the limit of thermodynamic equilibrium. Taking into account expressions (3.12) and (3.28) for the energy of the «ground state», the following expression can be written:

$$
E_{o s c}^{(0)}(\gamma)=\lim _{t \rightarrow+\infty}\left\{N_{0}^{-1}(t) \operatorname{Tr}_{x} \operatorname{Tr}_{\xi}\left[\hat{H}_{0} \rho_{s t c}\left(x, x^{\prime}, t \mid\{\xi\}\right)\right]\right\}
$$

where the operator:

$$
\hat{H}_{0}=\frac{1}{2}\left[-\partial_{x}^{2}+\Omega_{0}^{2} x^{2}\right],
$$

describes the Hamiltonian of $1 \mathrm{D}$ QHO without an environment.

Substituting (3.41) in (3.40) and after conducting simple calculations, we can find:

$$
E_{o s c}^{(0)}(\gamma)=\frac{1}{2}\left[1+K_{0}(\gamma)\right] \Omega_{0}
$$

where the following designations are made:

$$
K_{0}(\gamma)=\frac{1}{N_{0}(\gamma)} \int_{-\infty}^{+\infty} d \bar{u}_{1} \int_{0}^{+\infty} d \bar{u}_{2} \frac{1}{\sqrt{\bar{u}_{2}}}\left\{-1+\frac{\bar{u}_{1}^{2}+\bar{u}_{2}^{2}+\gamma^{2}}{2 \gamma \bar{u}_{2}}\right\} Q_{0}\left(\bar{u}_{1}, \bar{u}_{2}, \gamma\right), \quad \bar{u}_{1,2}=u_{1,2} / \lambda^{1 / 3} .
$$

In expression (3.43) the stationary solution $Q_{0}\left(\bar{u}_{1}, \bar{u}_{2}, \gamma\right)=\lim _{t \rightarrow+\infty} Q_{0}\left(\bar{u}_{1}, \bar{u}_{2}, t\right)$ is a scaling solution of equation (3.30) or (3.36) for the case where $\alpha=0$. Similarly, it is possible to calculate the average value of the energy of any excited state. In particular, the calculation of the energy level of the first excited state leads to the following expression:

$$
E_{o s c}^{(1)}(\gamma)=\frac{3}{2}\left[1+K_{1}(\gamma)\right] \Omega_{0},
$$


where

$$
K_{1}(\gamma)=\frac{1}{N_{1}(\gamma)} \int_{-\infty}^{+\infty} d \bar{u}_{1} \int_{0}^{+\infty} d \bar{u}_{2} \frac{1}{\bar{u}_{2}^{3 / 2}}\left\{-1+\frac{\bar{u}_{1}^{2}+\bar{u}_{2}^{2}+\gamma^{2}}{2 \gamma \bar{u}_{2}}\right\} Q_{1}\left(\bar{u}_{1}, \bar{u}_{2}, \gamma\right),
$$

in addition:

$$
N_{1}(\gamma)=\int_{-\infty}^{+\infty} d \bar{u}_{1} \int_{0}^{+\infty} d \bar{u}_{2} \frac{1}{\bar{u}_{2}^{3 / 2}} Q_{1}\left(\bar{u}_{1}, \bar{u}_{2}, \gamma\right)
$$

In expression (3.45) the stationary solution $Q_{1}\left(\bar{u}_{1}, \bar{u}_{2}, \gamma\right)=\lim _{t \rightarrow+\infty} Q_{1}\left(\bar{u}_{1}, \bar{u}_{2}, t\right)$ is a scaling solution of equation (3.36)) for the case where $\alpha=1$.

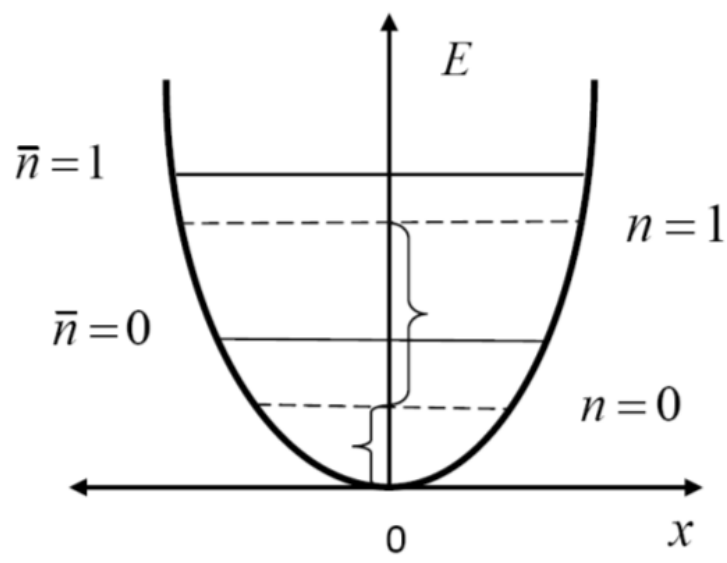

Fig. 1. The first two energy levels of quantum harmonic oscillator without of FE (see quantum numbers $\bar{n}=0,1, .$. ) and correspondingly with consideration of relaxation in the FE (see quantum numbers $n=0,1, .$. ).

As obviously follows from expressions (3.42)-(3.46), the relaxation effects lead to infringement of the principle of equidistance between the energy levels of a quantum harmonic oscillator Fig.1. In other words, relaxation of the quantum subsystem in fundamental environment leads to a shift of energy levels like the well-known Lamb shift.

\subsection{Spontaneous transitions between the energy levels of a quantum subsystem}

The question of stability of the energy levels of a quantum subsystem is very important. It is obvious that the answer to this question may be received after investigation of the problem of spontaneous transitions between the energy levels. Taking into account (3.4) and (3.8), we can write an expression for the probability of spontaneous transition between two different quantum states: 


$$
W_{n \rightarrow m}(\gamma)=\left|S_{n m}(\gamma)\right|^{2}, \quad S_{n m}(\gamma)=\lim _{t \rightarrow+\infty} \operatorname{Tr}_{x} \operatorname{Tr}_{\xi}\left\{\Psi_{s t c}(n|x, t|\{\xi\}) \Psi^{*}(m \mid x, t)\right\}
$$

where the wave function $\Psi(m \mid x, t)$ describes a pure state.

It is obvious that in the considered formulation of the problem there might occur transitions between any energy levels, including transitions from the "ground state» to any excited states. Using expression (3.47), we can calculate the spontaneous decay of every quantum state. In particular, if $w^{(0)}=1$ and $w^{(n)} \equiv 0$ for any $m \geq 1$, the probability of transition from the "ground state» to all other excited states may be calculated as follows:

$$
\Sigma^{0}(\gamma)=\sum_{m=1}\left|S_{0 m}(\gamma)\right|^{2}=\sum_{m} \Delta_{0 \rightarrow m}(\gamma)
$$

In (3.48) $\Sigma^{0}$ characterizes the population of the «ground state» in the limit of equilibrium thermodynamics. The first two nonzero probabilities of spontaneous transitions are calculated simply (see Appendix 4.2):

$$
\begin{aligned}
& \Delta_{0 \rightarrow 2}(\gamma)=\left|\int_{-\infty}^{+\infty} d \bar{u}_{1} \int_{0}^{+\infty} d \bar{u}_{2} \Theta\left(\bar{u}_{1}, \bar{u}_{2}, \gamma\right) \sigma_{0}\left(\bar{u}_{1}, \bar{u}_{2}, \gamma\right)\right|, \\
& \Delta_{2 \rightarrow 0}(\gamma)=\left|\int_{-\infty}^{+\infty} d \bar{u}_{1} \int_{0}^{+\infty} d \bar{u}_{2} \Theta\left(\bar{u}_{1}, \bar{u}_{2}, \gamma\right) \sigma_{2}\left(\bar{u}_{1}, \bar{u}_{2}, \gamma\right)\right|^{2},
\end{aligned}
$$

where

$$
\Theta\left(\bar{u}_{1}, \bar{u}_{2}, \gamma\right)=\frac{\sqrt{\gamma}}{\sqrt{\gamma-i \bar{u}_{1}+\bar{u}_{2}}}\left\{1-\frac{2 \gamma}{\gamma-i \bar{u}_{1}+\bar{u}_{2}}\right\} .
$$

Let us note that in expressions (3.48) and (3.49) the functions $\sigma_{0}\left(\bar{u}_{1}, \bar{u}_{2}, \gamma\right)$ and $\sigma_{2}\left(\bar{u}_{1}, \bar{u}_{2}, \gamma\right)$ are solutions of the equation:

$$
\partial_{t} \sigma_{n}\left(\bar{u}_{1}, \bar{u}_{2}, \gamma\right)=\left[\hat{L}-(1 / 2+n)\left(\bar{u}_{1}+\bar{u}_{2}\right)\right] \sigma_{n}\left(\bar{u}_{1}, \bar{u}_{2}, \gamma\right) .
$$

Comparing expressions (3.48) and (3.49) with taking into account the fact that equation (3.50) for a different number $n$ has different solutions, $\sigma_{n} \neq \sigma_{m}$ if $n \neq m$, we can conclude that the detailed balance of transitions between different quantum levels is violated, i. e. $\Delta_{0 \rightarrow 2} \neq \Delta_{2} \rightarrow 0$. Also, it is obvious that transitions between the quantum levels are possible if their parities are identical.

\subsection{Uncertainty relations, Weyl transformation and Wigner function for the ground state}

According to the Heisenberg uncertainty relations, the product of the coordinate and corresponding momentum of the quantum system cannot have arbitrarily small dispersions. This principle has been verified experimentally many times. However, at the present time for development of quantum technologies it is very important to find possibilities for overcoming this fundamental restriction. 
As is well-known, the dispersion of the operator $\hat{A}_{i}$ is determined by the following form:

$$
\Delta \hat{A}_{i}(t)=\left\{\operatorname{Tr}_{x}\left(\rho \hat{A}_{i}^{2}(t)\right)-\left[\operatorname{Tr}_{x}\left(\rho \hat{A}_{i}(t)\right)\right]^{2}\right\}
$$

In the considered case the dispersion of the operator at the arbitrary time $t$ in the "ground state» can be calculated by the following expression:

$$
\Delta \hat{A}_{i}(t)=N_{0}^{-1}(t)\left\{\operatorname{Tr}_{x} \operatorname{Tr}_{\xi}\left(\rho_{s t c}^{(0)} \hat{A}_{i}^{2}(t)\right)-\left[\operatorname{Tr}_{x} \operatorname{Tr}_{\xi}\left(\rho_{s t c}^{(0)} \hat{A}_{i}(t)\right)\right]^{2}\right\}
$$

Using expression (3.52), we can calculate the dispersions of operators, the coordinate, $\hat{x}$ and momentum, $\hat{p}$ correspondingly:

$$
\begin{aligned}
& \Delta \hat{x}(t)=\left\{\frac{1}{N_{0}(t)} \int_{-\infty}^{+\infty} d \bar{u}_{1} \int_{0}^{+\infty} d \bar{u}_{2} \frac{1}{\bar{u}_{2}^{3 / 2}} Q_{0}\left(\bar{u}_{1}, \bar{u}_{2}, \gamma\right)\right\}^{1 / 2}=\sqrt{\frac{1}{2 N_{0}(t)}} A_{x}(t), \\
& \Delta \hat{p}(t)=\left\{\frac{1}{N_{0}(t)} \int_{-\infty}^{+\infty} d \bar{u}_{1} \int_{0}^{+\infty} d \bar{u}_{2} \frac{\bar{u}_{1}^{2}+\bar{u}_{2}^{2}}{\bar{u}_{2}^{3 / 2}} Q_{0}\left(\bar{u}_{1}, \bar{u}_{2}, t\right)\right\}^{1 / 2}=\sqrt{\frac{1}{2 N_{0}(t)}} A_{p}(t) .
\end{aligned}
$$

The dispersions of operators at the moment of time $t_{0}$, when the interaction with the environment is not switched on, is described with the standard Heisenberg relation: $\left.\Delta \hat{x}(t) \Delta \hat{p}(t)\right|_{t=t_{0}}=1 / 2$. The uncertainty relation for the large interval of time when the united system approaches thermodynamic equilibrium can be represented in the form:

$$
\Delta \hat{x}_{s t} \Delta \hat{p}_{s t}=\lim _{t \rightarrow+\infty}[\Delta \hat{x}(t) \Delta \hat{p}(t)]=\frac{1}{2} \frac{A_{x}(\gamma) A_{p}(\gamma)}{N_{0}(\gamma)}
$$

where average values of operators $\hat{x}(\gamma)$ and $\hat{p}(\gamma)$ can be found from (3.53) and (3.54) in the limit $t \rightarrow+\infty$.

It is obvious that expressions for operator dispersions (3.53)-(3.54) are different from Heisenberg uncertainty relations and this difference can become essential at certain values of the interaction parameter $\gamma$. The last circumstance is very important since it allows controlling the fundamental uncertainty relations with the help of the $\gamma$ parameter.

Definition 4. We will refer to the expression:

$$
W_{s t c}(p, x, t ;\{\xi\})=\sum_{m=1}^{+\infty} w^{(m)} W_{s t c}(m \mid(p, x, t ;\{\xi\})),
$$

as stochastic Wigner function and, correspondingly, to $W_{s t c}(m \mid p, x, t ;\{\xi\})$ as partial stochastic Wigner function. In particular, for the partial stochastic Wigner function the following expression may be found: 


$$
W_{s t c}(m \mid p, x, t ;\{\xi\})=\frac{1}{2} \int_{-\infty}^{+\infty} e^{i p v} \Psi_{s t c}(m \mid x-v / 2, t ;\{\xi\}) \Psi_{s t c}^{*}(m \mid x+v / 2, t ;\{\xi\}) d v .
$$

Using the stochastic Wigner function, it is possible to calculate the mean values of the physical quantity, which corresponds to the operator $\hat{A}$ :

$$
\bar{A}(\lambda)=\lim _{t \rightarrow+\infty}\left\{\frac{1}{N(t)} \int_{-\infty}^{+\infty} d p \int_{-\infty}^{+\infty} d x \operatorname{Tr}_{\xi}\left[a(p, x, t ;\{\xi\}) \rho_{s t c}\left(x, x^{\prime}, t \mid\{\xi\}\right)\right]\right\},
$$

where the stochastic function $a(p, x, t ;\{\xi\})$ is defined with the help of a Weyl transformation of the operator $\hat{A}$ :

$$
a(p, x, t ;\{\xi\})=\int_{-\infty}^{+\infty} e^{i p v} \Psi_{s t c}(m \mid x-v / 2, t ;\{\xi\}) \hat{A} \Psi_{s t c}^{*}\left(m \mid x+v / 2, t ;\left\{\xi^{\prime}\right\}\right) d v .
$$

Now we can construct a Wigner function for the «ground state»:

$$
\begin{aligned}
& W^{(0)}(x, p, t)=\frac{1}{2} \operatorname{Tr}_{\xi}\left\{W_{s t c}(0 \mid x, p, t ;\{\xi\})\right\}= \\
& \frac{1}{\sqrt{\pi}} \int_{-\infty}^{+\infty} d \bar{u}_{1} \int_{0}^{+\infty} d \bar{u}_{2} \frac{1}{\sqrt{\bar{u}_{2}}} Q_{0}\left(\bar{u}_{1}, \bar{u}_{2}, t\right) \exp \left\{-\frac{\left(p-\bar{u}_{1} x\right)^{2}-\left(\bar{u}_{2} x\right)^{2}}{\bar{u}_{2}}\right\} .
\end{aligned}
$$

As one can see, function (3.61) describes distribution of the coordinate $x$ and momentum $p$ in the phase space. The Wigner stationary distribution function can be found in the limit of the stationary processes

$W^{(0)}(x, p, \gamma)=\lim _{t \rightarrow+\infty} W^{(0)}(x, p, t)$. It is important to note that in thesimilar to regular case after integration of the stochastic function $W_{s t c}(m \mid p, x, t ;\{\xi\})$ over the phase space; it is easy to get the normalization condition:

$$
\int_{-\infty}^{+\infty} d x \int_{-\infty}^{+\infty} d p W_{s t c}(m \mid x, p, t ;\{\xi\})=1
$$

Recall that for the Wigner function (3.61) in the general case the normalization condition of type (6.12) is not carried out.

\subsection{Conclusion}

Any quantum system resulting from the fact that all beings are immersed into a physical vacuum is an open system [1-3]. A crucially new approach to constructing the quantum mechanics of a closed non-relativistic system QS+FE has been developed recently by the authors of [5-8], based on the principle of local equivalence of Schrodinger representation. More precisely, it has been assumed that the evolution of a quantum system is such that it may be described by the Schrödinger equation on any small time interval, while the motion as a 
whole is described by a SDE for the wave function. However, in this case there arises a nonsimple problem of how to find a measure of the functional space, which is necessary for calculating the average values of various parameters of the physical system.

We have explored the possibility of building the non-relativistic quantum mechanics of a closed system QS+FE within the framework of one-dimensional QHO which has a random frequency. Mathematically, the problem is formulated in terms of SDE for a complex-valued probability process (3.1) defined in the extended space $R^{1} \otimes R_{\{\xi\}}$. The initial SDE for complex processes is reduced to the 1D Schrödinger equation for an autonomous oscillator on a random space-time continuum (3.6). For this purpose the complex SDE of Langevin type has been used. In the case when random fluctuations of $\mathrm{FE}$ are described by the white noise correlation function model, the Fokker-Plank equation for conditional probability of fields is obtained (3.22)-(3.23) using two real-valued SDE for fields (3.20). With the help of solutions of this equation, a measure of the functional space $R_{\{\xi\}}$ is constructed (3.27) on infinitely small time intervals (3.24).In the context of the developed approach representation of the stochastic density matrix is introduced, which allows perform an exact computation scheme of physical parameters of $\mathrm{QHO}$ (of a quantum subsystem) and also of fundamental environment after relaxation under the influence of QS. The analytic formulas for energies of the "ground state» and for the first excited state with consideration of shift (like the Lamb shift) are obtained. The spontaneous transitions between various energy levels were calculated analytically and violation of symmetry between elementary transitions up and down, including spontaneous decay of the "ground state», was proved. The important results of the work are the calculation of expressions for uncertainty relations and Wigner function for a quantum subsystem strongly interacting with the environment.

Finally, it is important to note that the developed approach is more realistic because it takes into account the shifts of energy levels, spontaneous transitions between the energy levels and many other things which are inherent to real quantum systems. The further development of the considered formalism in application to exactly solvable manydimensional models can essentially extend our understanding of the quantum world and lead us to new nontrivial discoveries.

\section{Appendix}

\subsection{Appendix 1}

Theorem. Let us consider a set of random processes $\xi \equiv\left\{\xi_{1}, \xi_{2}, \ldots \xi_{n}\right\}$ satisfying the set of SDE:

$$
\dot{\xi}_{i}=a_{i}(\boldsymbol{\xi}, t)+\sum_{j=1}^{n} b_{j i}(\boldsymbol{\xi}, t) f_{j}(t), \quad i=1,2, \ldots . n,
$$

where

$$
\left\langle f_{i}(t)\right\rangle=0, \quad\left\langle f_{i}(t) f_{j}\left(t^{\prime}\right)\right\rangle=\delta_{i j} \delta\left(t-t^{\prime}\right),
$$

so that the Fokker-Planck equation for the conditional transition probability density:

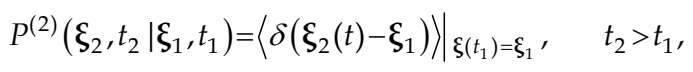


is given by the equation:

$$
\partial_{t} P^{(2)}=-\sum_{i=1}^{n} \frac{\partial}{\partial \xi_{i}}\left(a_{i} P^{(2)}\right)+\sum_{i j l k} \frac{\partial}{\partial \xi_{i}}\left(b_{l i} \frac{\partial}{\partial \xi_{k}}\left(b_{k j} P^{(2)}\right)\right) \equiv \hat{L}^{(n)}(\xi) P^{(2)} .
$$

$\xi_{i}$ are assumed to be Markovian processes and satisfy the condition $\boldsymbol{\xi}\left(t_{0}\right) \equiv \boldsymbol{\xi}_{0}$. At the same time function (4.1.2) gives their exhaustive description:

$$
P^{(n)}\left(\xi_{n}, t_{n} ; \ldots \xi_{1}, t_{1} ; \xi_{0}, t_{0}\right)=P^{(2)}\left(\xi_{n}, t_{n} ; \xi_{n-1}, t_{n-1}\right) \ldots P^{(2)}\left(\xi_{1}, t_{1} ; \xi_{0}, t_{0}\right),
$$

where $P^{(n)}$ is the density of the probability that the trajectory $\xi(t)$ would pass through the sequence of intervals $\left[\boldsymbol{\xi}_{1}, \boldsymbol{\xi}_{1}+d \boldsymbol{\xi}_{1}\right], \ldots .\left[\boldsymbol{\xi}_{n}, \boldsymbol{\xi}_{n}+d \boldsymbol{\xi}_{n}\right]$ at the subsequent moments of time $t_{1}<t_{2} \ldots<t_{n}$, respectively. Under these assumptions we can obtain the following representation for an averaging procedure:

$$
\left\langle\exp \left\{-\int_{t_{0}}^{t} V_{1}(\xi(t), \xi(\tau)) d \tau-V_{2}(\boldsymbol{\xi}(t))\right\}\right\rangle=\int d \xi e^{-V_{2}(\xi, t)} Q\left(\xi, \xi^{\prime}, t\right),
$$

where $d \boldsymbol{\xi}=d \xi_{1} \ldots d \boldsymbol{\xi}_{n}$ and the function $Q\left(\xi, \xi^{\prime}, t\right)$ is a solution of the following parabolic equation:

$$
\partial_{t} Q=\left[\hat{L}^{(n)}(\xi)-V_{1}\left(\xi, \xi^{\prime}, t\right)\right] Q,
$$

which satisfies the following initial and boundary conditions:

$$
Q\left(\xi, \xi^{\prime}, t\right) \underset{t \rightarrow t_{0}}{\rightarrow} \delta\left(t-t_{0}\right), \quad Q\left(\xi, \xi^{\prime}, t\right)_{|| \xi|| \rightarrow \infty}^{\rightarrow} 0,
$$

where ||$. . . \mid$ is a norm in $R^{n}$.

Proof. The proof is performed formally under the assumption that all the manipulations are legal. We will expand into the Taylor series the quantity under the averaging in the lefthand side of (4.1.5):

$$
I(t)=\sum_{n=0}^{+\infty} \frac{(-1)^{n}}{n !} \mu_{n}(t), \quad m=0,1, \ldots n
$$

where

$$
\begin{aligned}
& \mu_{n}(t)=\left\langle\left\{\int_{t_{0}}^{t} V_{1}(\tau) d \tau+V_{1}(t)\right\}^{n}\right\rangle=\sum_{m=0}^{+\infty} \frac{n !}{m !(n-m) !}\left\langle V_{2}^{n-m}(t)\left\{\int_{t_{0}}^{t} V_{1}(\tau) d \tau\right\}\right\rangle= \\
& \sum_{m=0}^{+\infty} \frac{n !}{m !(n-m) !}\left\langle V_{2}^{n-m}(t) \int_{t_{0}}^{t} d \tau_{m} \int_{t_{0}}^{\tau_{m}} d \tau_{m-1} \ldots \int_{t_{0}}^{\tau_{2}} d \tau_{1} V_{1}\left(\tau_{m}\right) V_{1}\left(\tau_{m-1}\right) \ldots V_{1}\left(\tau_{2}\right)\right\rangle .
\end{aligned}
$$

The designations $V_{1}(\tau) \equiv V_{1}(\xi(\tau), \xi(t))$ and $V_{2}(t) \equiv V_{2}(\xi(t))$ are introduced in (4.1.9) for brevity. Using the Fubini theorem, we can represent the averaging procedure in (4.1.9) as integration with the weight $P^{(n)}$ from (4.1.4): 


$$
\begin{aligned}
& \left\langle V_{2}^{n-m}(t) \int_{t_{0}}^{t} d \tau_{m} \int_{t_{0}}^{\tau_{m}} d \tau_{m-1} \ldots \int_{t_{0}}^{\tau_{2}} d \tau_{1} V_{1}\left(\tau_{m}\right) V_{1}\left(\tau_{m-1}\right) \ldots V_{1}\left(\tau_{2}\right)\right\rangle=\int d \boldsymbol{\int} d \boldsymbol{\xi}_{m} \ldots \int d \boldsymbol{\xi}_{1} \int_{t_{0}}^{\tau_{m-1}} d \tau_{m} \ldots \int_{t_{0}}^{\tau_{2}} d \tau_{1} \times \\
& P^{(2)}\left(\boldsymbol{\xi}, t \mid \boldsymbol{\xi}_{m}, \tau_{m}\right) P^{(2)}\left(\boldsymbol{\xi}_{m}, \tau_{m} \mid \boldsymbol{\xi}_{m-1}, \tau_{m-1}\right) \ldots P^{(2)}\left(\boldsymbol{\xi}_{2}, \tau_{2} \mid \boldsymbol{\xi}_{1}, \tau_{1}\right) V_{2}^{n-m}(\boldsymbol{\xi}) V_{1}\left(\boldsymbol{\xi}_{m} \boldsymbol{\xi}\right) \ldots V_{1}\left(\boldsymbol{\xi}_{1} \boldsymbol{\xi}\right) .
\end{aligned}
$$

Changing, where it is necessary, the order of integration, we can obtain the following representation for the $n$-th moment:

$$
\mu_{n}(t)=\sum_{m=0}^{n} \frac{n !}{(n-m) !} \int d \xi V_{2}^{n-m}(\xi) Q_{m}\left(\xi, \boldsymbol{\xi}^{\prime}, t\right)
$$

where the countable set of functions $Q_{m}\left(\mathcal{\xi}, \boldsymbol{\zeta}^{\prime}, t\right)$ is determined from the recurrence relations:

$$
Q_{m}\left(\boldsymbol{\xi}, \boldsymbol{\xi}^{\prime}, t\right)=\int_{t_{0}}^{t} d \tau \int d \zeta V_{2}^{n-m}(\boldsymbol{\xi}) P^{(2)}\left(\boldsymbol{\xi}^{\prime}, t \mid \zeta, \tau\right) V_{1}\left(\zeta, \boldsymbol{\zeta}^{\prime}\right) Q_{m-1}\left(\boldsymbol{\zeta}, \boldsymbol{\zeta}^{\prime}, \tau\right)
$$

where

$$
Q_{0}\left(\mathcal{\zeta}, \mathcal{\zeta}^{\prime}, t\right)=P^{(2)}\left(\xi, t \mid \xi_{0}, t_{0}\right),
$$

i.e. the function $Q_{0}$ is, in fact, independent of $\xi^{\prime}$. Upon substitution of (4.1.10) into (4.1.8) we insert the summation procedure under the integration sign and then, changing the order of double summation, get the expression:

$$
I(t)=\int d \xi e^{-V_{2}(\xi, t)} Q\left(\xi, \xi^{\prime}, t\right)
$$

where

$$
Q\left(\xi, \xi^{\prime}, t\right)=\sum_{n=0}^{\infty}(-1)^{n} Q_{n}\left(\xi, \xi^{\prime}, t\right)
$$

The representation (4.1.5) is thus obtained.

It remains to prove that the function $Q$ from (4.1.13) is a solution of the problem (4.1.6) (4.1.7). Using (4.1.14) and (4.1.11) we can easily show that $Q$ satisfies the integral equation:

$$
Q\left(\xi, \xi^{\prime}, t\right)+\int_{t_{0}}^{t} d \tau \int d \zeta P^{(2)}(\xi, t \mid \zeta, \tau) V_{1}\left(\zeta, \xi^{\prime}, \tau\right) Q\left(\zeta, \xi^{\prime}, \tau\right)=Q_{0}(\xi, t)
$$

Taking into account the fact that $Q_{0}$ satisfies equation (4.1.3) with the initial and border conditions (4.1.7) and also that it is an integrable function, it is easy to deduce from equation (4.1.16) that the $Q$ function coincides with the solution of the problem (4.1.6)-(4.1.7). Thus, the theorem is proved.

\subsection{Appendix 2}

Let us consider the bilinear form: 


$$
\mathfrak{I}(n, m, \mid x, t ;\{\xi\})=\Psi_{s t c}(n|x, t|\{\xi\}) \Psi^{*}(m \mid x, t),
$$

which can be represented,taking into account expressions (3.4) and (3.8), by the following form:

$$
\begin{gathered}
\mathfrak{I}(n, m, \mid x, t ;\{\xi\})=\left(\frac{\Omega_{0} / \pi}{2^{n+m} n ! m !}\right)^{1 / 2}\left[u_{2}(t)\right]^{-n / 2} \exp \left\{-\left(\frac{1}{2}+n\right) \int_{t_{0}}^{t}\left[u_{1}\left(t^{\prime}\right)+u_{2}\left(t^{\prime}\right)\right] d t^{\prime}\right. \\
\left.-\frac{1}{2}\left[-i u_{1}(t)+u_{2}(t)+\Omega_{0}\right] x^{2}\right\} H_{n}\left(\sqrt{u_{2}(t)} x\right) H_{m}\left(\sqrt{\Omega_{0}} x\right) .
\end{gathered}
$$

After conducting functional integration of the expression $\mathfrak{I}(n, m, \mid x, t ;\{\xi\})$ by the generalized Feynman-Kac formula (see Appendix 4.1), it is possible to find:

$$
\begin{gathered}
\Im(n, m, \mid x, t ;\{\xi\})=\operatorname{Tr}_{\{\xi\}}\{\Im(n, m, \mid x, t ;\{\xi\})\}=\left(\frac{\Omega_{0} / \pi}{2^{n+m} n ! m !}\right)^{1 / 2} H_{m}\left(\sqrt{\Omega_{0}} x\right) \int_{-\infty}^{+\infty} d \bar{u}_{1} \int_{0}^{+\infty} d \bar{u}_{2} \times \\
{\left[\bar{u}_{2}\right]^{-n / 2} \exp \left\{-\frac{1}{2}\left[-i u_{1}(t)+u_{2}(t)+\Omega_{0}\right] x^{2}\right\} H_{n}\left(\sqrt{u_{2}} x\right) \mathrm{X}_{n}\left(u_{1}, u_{2}, t\right),}
\end{gathered}
$$

where $\mathrm{X}_{n}\left(u_{1}, u_{2}, t\right)$ is a solution of the complex equation:

$$
\partial_{t} \mathrm{X}_{n}\left(u_{1}, u_{2}, t\right)=\left[\hat{L}-(1 / 2+n)\left(u_{1}+i u_{2}\right)\right] \mathrm{X}_{n}\left(u_{1}, u_{2}, t\right) .
$$

The solution of equation (4.4) is useful to represent in the following form:

$$
\mathrm{X}_{n}\left(u_{1}, u_{2}, t\right)=\sigma_{n}\left(u_{1}, u_{2}, t\right)+i \chi_{n}\left(u_{1}, u_{2}, t\right)
$$

By substituting (4.2.5) into equation (4.2.4), it is possible to find the following two real-value equations for the real and complex parts of solution:

$$
\begin{aligned}
& \partial_{t} \sigma_{n}\left(u_{1}, u_{2}, t\right)=\left[\hat{L}-(1 / 2+n) u_{1}\right] \sigma_{n}\left(u_{1}, u_{2}, t\right)+(1 / 2+n) u_{2} \chi_{n}\left(u_{1}, u_{2}, t\right), \\
& \partial_{t} \chi_{n}\left(u_{1}, u_{2}, t\right)=\left[\hat{L}-(1 / 2+n) u_{1}\right] \chi_{n}\left(u_{1}, u_{2}, t\right)-(1 / 2+n) u_{2} \sigma_{n}\left(u_{1}, u_{2}, t\right) .
\end{aligned}
$$

The system of equations is symmetric in regard to the replacements: $\sigma_{n} \rightarrow-\chi_{n}$ and $\chi_{n} \rightarrow-\sigma_{n}$. In other words, it means that for the solution $\sigma_{n}\left(u_{1}, u_{2}, t\right)$ it is possible to write the following equation:

$$
\partial_{t} \sigma_{n}\left(u_{1}, u_{2}, t\right)=\left[\hat{L}-(1 / 2+n)\left(u_{1}+u_{2}\right)\right] \sigma_{n}\left(u_{1}, u_{2}, t\right) .
$$

Accordingly, for a complex solution $\mathrm{X}_{n}\left(u_{1}, u_{2}, t\right)$ we can write the expression:

$$
\mathrm{X}_{n}\left(u_{1}, u_{2}, t\right)=(1-i) \sigma_{n}\left(u_{1}, u_{2}, t\right)=-(1-i) \chi_{n}\left(u_{1}, u_{2}, t\right) .
$$

Now it is possible to pass to the calculation of the amplitude of transition between different quantum states. For simplicity we will compute the first two probabilities of transitions: 
$\Delta_{0 \rightarrow 2}$ and $\Delta_{2 \rightarrow 0}$. Integrating the expression $\left.\mathfrak{J}(0,2, \mid x, t\}\right)$ over $x$ with taking into account result (4.2.8), it is easy to find:

$$
\left.S_{02}(\gamma)=\lim _{t \rightarrow+\infty} \int_{-\infty}^{+\infty} \Im(0,2, \mid x, t\}\right) d x=\sqrt{\gamma} \int_{-\infty}^{+\infty} d \bar{u}_{1} \int_{0}^{+\infty} d \bar{u}_{2} \Theta\left(\bar{u}_{1}, \bar{u}_{2}, \gamma\right) \sigma_{0}\left(\bar{u}_{1}, \bar{u}_{2}, \gamma\right),
$$

where $\sigma_{0}\left(\bar{u}_{1}, \bar{u}_{2}, \gamma\right)$ is the scaled solution of equation (4.2.7) in the limit $t \rightarrow+\infty$, in addition:

$$
\Theta\left(\bar{u}_{1}, \bar{u}_{2}, \gamma\right)=\left(\gamma-i \bar{u}_{1}+\bar{u}_{2}\right)^{-1 / 2}\left[1-2 \gamma\left(\gamma-i \bar{u}_{1}+\bar{u}_{2}\right)^{-1}\right] .
$$

In a similar way it is possible to calculate the transition matrix element $S_{20}(\gamma)$ :

$$
\left.S_{20}(\gamma)=\lim _{t \rightarrow+\infty} \int_{-\infty}^{+\infty} \Im(2,0, \mid x, t\}\right) d x=\sqrt{\gamma} \int_{-\infty}^{+\infty} d \bar{u}_{1} \int_{0}^{+\infty} d \bar{u}_{2} \Theta\left(\bar{u}_{1}, \bar{u}_{2}, \gamma\right) \sigma_{2}\left(\bar{u}_{1}, \bar{u}_{2}, \gamma\right)
$$

As follows from expressions (4.2.9), (4.2.10) and (4.2.11), in the general case $S_{02}(\gamma) \neq S_{20}(\gamma)$.

\section{Acknowledgment}

This Chapter was prepared and written with the kind help of Ms. Elena Pershina.

\section{References}

[1] Davtyan, O.K.; Theory of gravitational-inertial field of universe. IV. The universe and the microcosm. Ann. Phys. 36, 227-237 (1979)

[2] Davtyan, O. K. \& Karamyan, G.G.; Theories of Inertial Fields and of Quantum Correlations. Academy of Science of Arm. SSR, Yerevan (1987)

[3] Milonni, P. W.; The Quantum Vacuum. Academic Press, San Diego (1994)

[4] Bogdanov, A. V., Gevorkyan, A.S. \& Grigoryan, A.G.; Random Motion of Quantum Harmonic Oscillator. Thermodynamics of Nonrelativistic Vacuum. AMS/IP Studies in Advanced Mathematics, 13, 81-111, (1999).

[5] Bogdanov, A. V., Gevorkyan, A.S. \& Udalov, A. A.; Thermodynamics of homogeneous and nonhomogeneous spaces in framework of representation by stochastic density matrix, XXIII Internat. Colloquium on Group theoretical methods in Physics, Vol. 2, pp. 419-429, Eds by A.N. Sisakian, A.N. Pogosyan \& L.G. Mardoyan, (Dubna 2000): JINR.

[6] Gevorkyan, A.S.; Exactly solvable models of stochastic quantum mechanics within the framework of Langevin-Schrödinger type equation. In: Barsegian, G. \& Begehr, H. (eds.) Analysis and Applications, p. 415. NATO Science Publications, Brussels (2004)

[7] Gevorkyan, A.S.; Exactly constructed model of quantum mechanics with random environment. Phys. Atom. Nucl. 73(N2), 311-319 (2010)

[8] Gevorkyan, A.S.; Nonrelativistic quantum mechanics with fundamental environment, Foundation of Physics 41, 509-515 (2011). DOI 10.1007/s100701-010-9446-y. 
[9] Gevorkyan, A.S., Burdik, C. \& Oganesyan, K.B.; Quantum harmonic oscillator subjected to quantum vacuum fluctuations, Proceedingsof AIP Conference N 1232, pp. 251-258, ISBN 978-0-7354-07777-0, QTRF-5, Vaxjo Sweden July 16-20 (2010)

[10] Klyatskin, V.I, Statistical Description of Dynamical Systems with Fluctuating Parameters (Nauka, Moscow, 1975) [in Russian].

[11] Von Neumann, J.; Mathematical Foundations of Quantum Mechanics. Princeton University Press, Princeton (1955)

[12] Encyclopedia of Mathematical Physics (The Big Soviet Encyclopedia, Moscow, 1998) [in Russian]

[13] Gevorkyan, A. S., Karamyana, G. G. \& Sargsyan, R. Sh.; Quantum-mechanical channel of interactions between macroscopic systems, Proceedings of AIP Conference N 1232, pp 267-274, ISBN 978-0-7354-07777-0, QTRF-5 Vaxjo Sweden, July 16-20,(2010)

[14] Girardi, G.C., Rimini, A.\&Weber, T.; Unified dynamics for microscopic and macroscopic systems. Phys. Rev. D 34, 470-477 (1986)

[15] Itano, W.M., Heinzen, D. J., Bellinger, J. J. \& Wineland, D. J.; Quantum Zeno effect, Phys. Rev. A41, 2295 (1990)

[16] Percival, C.; Quantum State Diffusion(Cambridge Univ., Cambridge, 2000)

[17] Gorini, V., Kossakowski, A. \& Sudarshan, C. G.; Completely positive dynamical semigroups of N - level systems, J.Math. Phys.17, 821 (1976).

[18] Lindblad, G.; On the generators of quantum dynamical semi-groups, Commun. Math. Phys.48, 119 (1976)

[19] Weiss, U.; Quantum Dissipative Systems (World Sci., Singapore, 1993)

[20] Gisin, N \& Percival, I.C.; The quantum-state diffusion model applied to open system, J. Phys. A25, 5677 (1992)

[21] Feynman, R.P. \& Vernon, F.L., The theory of a general quantum system interacting with a linear dissipative system, Jr., Ann. Phys. (N.Y.) 24, 118 (1963)

[22] Dekker, H.; Classical and Quantum Mechanics of the Damped Oscillator, Phys. Rep. 80, 1 (1981)

[23] Caldeira, A. O. \& Leggett, A.J.; Path integral approach to quantum Brownian motion, Physica A121, 587 (1983); Quantum tunneling in dissipative system, Ann. Phys. (N.Y.) 149, 374 (1983); Influence and damping on quantum interference: An exact solvable model, Phys. Rev. A31, 1059 (1985)

[24] Jo'os, E. \& Zeh, H. D.; The emergence of classical properties through interaction with environment, Z. Phys. B 59, 223 (1985)

[25] Paz, J. P. \& Zurek, W.H.; Environment-induced decoherence, classically and consistency of quantum histories, Phys. Rev. D48, 2728 (1993)

[26] Unruh, W. G. \& Zurek, W. H.; Reduction of the wavepacket in quantum Brownian motion, Phys. Rev. D 40, 1071 (1989)

[27] Zurek, W. H.; Decoherence, chaos, quantum-classical correspondence, and the algorithmic arrow of time, Phys. Scripta 76, 186 (1998)

[28] Baz', A.N., Zel'dovich, Ya.B. \& Perelomov, A.M.; Scattering Reactions and Decays in Nonrelativistic Quantum Mechanics(Nauka, Moscow, 1971) [in Russian]

[29] Lifshitz, I.M., Gredeskul, S.A. \& Pastur, L.P.; Introduction to the Theory of Nonregular Systems (Nauka, Moscow, 1982) [in Russian]

[30] Gardiner, C.W.; Handbook of Stochastic Methods for Physics, Chemistry and Natural Sciences (Springer, Berlin, New York, Tokyo, 1985) 
[31] Kudryavtsev, L. D.; Fubini theorem, Encyclopedia of Mathematics, Spinger 2001, ISBN 978-155608104. 


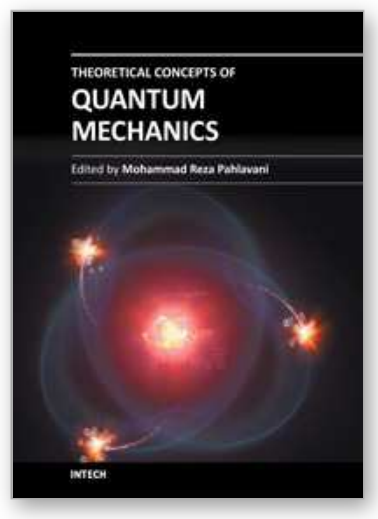

\author{
Theoretical Concepts of Quantum Mechanics \\ Edited by Prof. Mohammad Reza Pahlavani
}

ISBN 978-953-51-0088-1

Hard cover, 598 pages

Publisher InTech

Published online 24, February, 2012

Published in print edition February, 2012

Quantum theory as a scientific revolution profoundly influenced human thought about the universe and governed forces of nature. Perhaps the historical development of quantum mechanics mimics the history of human scientific struggles from their beginning. This book, which brought together an international community of invited authors, represents a rich account of foundation, scientific history of quantum mechanics, relativistic quantum mechanics and field theory, and different methods to solve the Schrodinger equation. We wish for this collected volume to become an important reference for students and researchers.

\title{
How to reference
}

In order to correctly reference this scholarly work, feel free to copy and paste the following:

Ashot S. Gevorkyan (2012). Nonrelativistic Quantum Mechanics with Fundamental Environment, Theoretical Concepts of Quantum Mechanics, Prof. Mohammad Reza Pahlavani (Ed.), ISBN: 978-953-51-0088-1, InTech, Available from: http://www.intechopen.com/books/theoretical-concepts-of-quantum-mechanics/nonrelativisticquantum-mechanics-with-fundamental-environment

\section{INTECH}

open science | open minds

\section{InTech Europe}

University Campus STeP Ri

Slavka Krautzeka 83/A

51000 Rijeka, Croatia

Phone: +385 (51) 770447

Fax: +385 (51) 686166

www.intechopen.com

\section{InTech China}

Unit 405, Office Block, Hotel Equatorial Shanghai

No.65, Yan An Road (West), Shanghai, 200040, China

中国上海市延安西路65号上海国际贵都大饭店办公楼 405 单元

Phone: +86-21-62489820

Fax: +86-21-62489821 
(C) 2012 The Author(s). Licensee IntechOpen. This is an open access article distributed under the terms of the Creative Commons Attribution 3.0 License, which permits unrestricted use, distribution, and reproduction in any medium, provided the original work is properly cited. 